\title{
Cellular Transplantation for the Treatment of Heart Failure. State of the Art
}

\author{
Marcio Scorsin, Luiz Cesar Guarita Souza \\ Curitiba, PR
}

The technique of transplanting muscle cells into the myocardium has been developed over the past few years in different animal models, with the aim of repopulating the impaired cardiac muscle with functional tissue. A clinical trial is now underway in France to assess the real benefit of this new therapy.

Heart failure is becoming a major public health problem, and considering the increased longevity rates of the world population, it is expected to be the prevailing cardiac disease of the next century. This is basically caused by the progress in cardiology, which has reduced mortality from acute myocardial infarction, and has been interpreted as an "ironic failure of success". When this disease becomes refractory to pharmacological therapy, available surgical treatments appear, the most radical being the replacement of the entire failing organ, as in cardiac transplantation. However, this technique has some limitations inherent to the procedure, such as organ shortage and graft vasculopathy. We must consider the fact that this treatment option cannot be proposed to all potential candidates. The intrathoracic implantation of circulatory assist devices is still primarily considered as a bridge to transplantation in the most critically ill patients. Another interesting option is xenotransplantation, which is still confined to the experimental stage, in particular because of the difficulty in adequately addressing major safety issues.

Among the less aggressive surgical techniques proposed ${ }^{1}$, the great majority attempt to change the geometry and the dilatation of the left ventricle. To maintain cardiac output, the left ventricle increases its volume loosing its normal oval shape and acquiring a rounded aspect. Reshaping the left ventricle could be very attractive considering the Starling law, but it does not correct the underlying disease that triggered the remodeling process, that is cardiomyocyte death, often responsible for the development of progressive congestive heart failure.

Clínica Cardiológyca C. Costantini - Curitiba

Mailing address: Marcio Scorsin - Clínica Cardiológyca C. Costantini - Rua Pedro Collere, 890 - Curitiba, PR - 80320-320 - e-mail: clinicard@bsi.com.br

Recebido para publicação em 12/2/01

Aceito em 28/3/01
With the objective of increasing ventricular mass, Leriche and Fontaine ${ }^{2}$ proposed, in an experimental study to wrap the heart with a skeletal muscle, the latissimus dorsi. Five decades later dynamic cardiomyoplasty was born ${ }^{3}$ and was used as an option for to restoring myocardial contractility. However, it has yielded rather inconsistent results basically owing to skeletal muscle atrophy.

In this setting, over these past years, cellular transplantation has emerged as an attractive alternate therapy for severe heart failure. This approach was encouraged by the recognition that normal myocardium could be successfully colonized by a variety of contractile cells. The engraftment of cardiomyocytes has been demonstrated by injecting cells taken from transgenic mice expressing the $\beta$-galactosidase gene ${ }^{4}$, which could then be identified by specific staining. Likewise, the presence of allogeneic-dystrophin-positive cells in the hearts of dogs suffering from Duchenne muscular dystrophy, which is characterized by a lack of dystrophin, has brought additional evidence for the capacity of transplanted cells to be harbored and integrated into the recipient myocardium ${ }^{5}$ within the cardiac tissue, as demonstrated by the formation of intercalated discs between host and recipient cells ${ }^{4}$. Finally, fetal cardiomyocytes have been shown to be able to survive in the bordering zone of a myocardial infarction ${ }^{6}$ opening the possibility of colonizing ischemic myocardium.

In a second step, different studies have assessed whether cellular transplantation could effectively improve the function of ischemically diseased myocardium. A positive answer to this question has been achieved by Li and coworkers ${ }^{7}$ who have shown, in a rat model of cryoinjury-induced myocardial infarction, that the intramyocardial injection of fetal cardiomyocytes improved systolic and diastolic function up to 2 months after transplantation, as assessed by ex vivo Langendorff perfusion studies. To this end, Scorsin and coworkers ${ }^{8}$ developed a protocol of echocardiography allowing an accurate analysis of two-dimensional echocardiographic images recorded in these fast-beating animals (approximately 300 beats/minute). In a reperfused infarcted area in rats, fetal cardiomyocytes were intramyocardially implanted, and, 1 month later, function was found to be significantly improved (as reflected by higher values in ejection fraction and cardiac output) in transplanted animals, when compared with controls that had only received an equivalent volume of culture medium alone. 
Another important issue was to determine whether functional benefits of cellular transplantation, documented in a model of regional ischemia, could be extended to the setting of global heart failure. To this end, in a mouse model of anthracycline-induced toxic cardiomyopathy ${ }^{9}$, it was possible to show that injected fetal cardiomyocytes also improved cardiac function 1 month after transplantation, as compared with control nontransplanted animals.

The advantage of fetal cardiomyocytes as cellular grafts is their capacity of entering the cell cycle and developing connections with host cells, an essential condition for functional improvement. Nevertheless, availability issues, ethical problems related to the fetal source of these cells, and, mainly, the necessity of immunosuppressive therapy are real limitations for a potential clinical application of this allograft technique. Because of the previously mentioned drawbacks associated with the use of fetal cells, the search for alternate cellular types was then refocused on the idea of a clinical use of autografts. Among the cells with the greatest therapeutic potential, bone marrow stromal cells and skeletal myoblasts appear to be the most promising.

\section{Bone marrow cells}

Bone marrow contains multipotential progenitor cells (mesenchymal stem cells) that are in an undifferentiated state with a highly proliferative capacity. With the use of in vitro chemical induction, they can differentiate into bone, tendon, fat, and muscle. In a very complex culture procedure ${ }^{10}$ in which cells were exposed to 5 -azacytidine, $30 \%$ of them were able to change their initial stem form into cardiomyocytelike cells, including the presence of intercalated disks and myotubes. Some studies showed ${ }^{11}$ that stromal bone marrow stem cells, without in vitro muscular differentiation, transplanted in a normal myocardium could undergo " milieu dependant" differentiation and express cardiogenic phenotype. However, in a cryoinjury model of myocardial infarction in rats ${ }^{12}$, only previously in vitro chemically induced muscular differentiated cells were able to improve function 2 months after transplantation. Despite this very interesting and promising option to restore myocardial viability, use of bone marrow stem cells raises two important concerns : 1) The multiplication potentiality and production of a very large number of differentiated cells in vitro has not yet been demonstrated. Cell duplication and reproduction is the first condition to fully recolonize the diseased myocardium and thus improve ventricular function; 2) A risk is present of developing other types of tissue, especially if undifferentiated stem cells are used. If the hypothesis that stromal cells can undergo phenotypic changes according to the histologic characteristic of the host tissue is legitimate, it sounds logical to imagine that they could be differentiated into fibroblasts instead of cardiomyocytes when transplanted in a fibrotic scar, consequently reducing the therapeutic spectrum of this type of procedure.

\section{Skeletal myoblasts (satellite cells)}

The precursor of skeletal muscle fiber, the myoblast ${ }^{13}$, is present in adult mammals as a quiescent cell and may activate, proliferate, and differentiate upon muscle injury (in vivo) or following tissue dissociation (in vitro) in culture.

Working on primary myoblast transplantations in a cryoinjury model of myocardial infarction in dogs, Chiu et al. ${ }^{14}$ were able to characterize the donor cells in the myocardium 14 weeks after injection. In this study, they identified myoblasts showing a "milieu dependant" differentiation, these cells having lost their characteristic skeletal morphology, acquiring a cardiac-like phenotype. Murry et al. ${ }^{15}$ observed the formation of myotubes and skeletal muscle fibers within the cardiac tissue, but they were unable to identify cardiacspecific markers within the tissue formed by the injection of myoblasts. More recently, Taylor et al. ${ }^{16}$ obtained functional improvement, as assessed by sonomicrometry, by transplanting myoblasts in a cryoinfarcted rabbit heart model where skeletal muscle cells were found within the scar tissue following transplantation. Of note is the fact that functional improvement was only seen in those animals in which implanted cells were histologically identified, thereby providing strong evidence for a causal relationship between the presence of engrafted cells and the functional outcome. In a myocardial infarction model in rats created by coronary artery ligation, Scorsin et al. ${ }^{17}$ documented a significant improvement in function, primarily manifested as a limitation of the postinfarction ventricular remodeling, compared with nontransplanted controls. Transplanted cells could be identified by a positive staining for embryonic myosin-heavy chain (which is specific for skeletal myoblasts), and no gap-junctions were detected between transplanted cells as expressed by the negative staining of the connexin-43, which is consistent with the study by Murry et al. Performing the same experiment ${ }^{17}$, Scorsin et al. ${ }^{17}$ compared the functional outcome between fetal cardiomyocytes and skeletal myoblasts, the latter being as effective as fetal cardiomyocytes in improving the function of the infarcted myocardium. This was a very important datum considering that the fetal cardiomyocyte remains as the standard cell in terms of restoring cardiac function. The fact that myoblasts were functionally equivalent opened the way for using them as autografts in an attempt to restore myocardial viability in humans.

After many years of preclinical research studies, the French health authorities have approved the undertaking of a phase 1 trial concerning myoblast transplantation in patients with severe heart failure. The results obtained in the first clinical case ${ }^{18}$ were very promising. After transplanting 800 million cells (Fig. 1) in an inferior wall of a 2year-old myocardial infarction scar (nonreversible akinetic by dobutamine echocardiography, with an absence of viability confirmed by positron emission tomography -PET), the akinetic wall became contractile (echocardiographic positive response by 1 grade to dobutamine stress), improving the left ventricular ejection fraction from 20 to $38 \%$ with a 


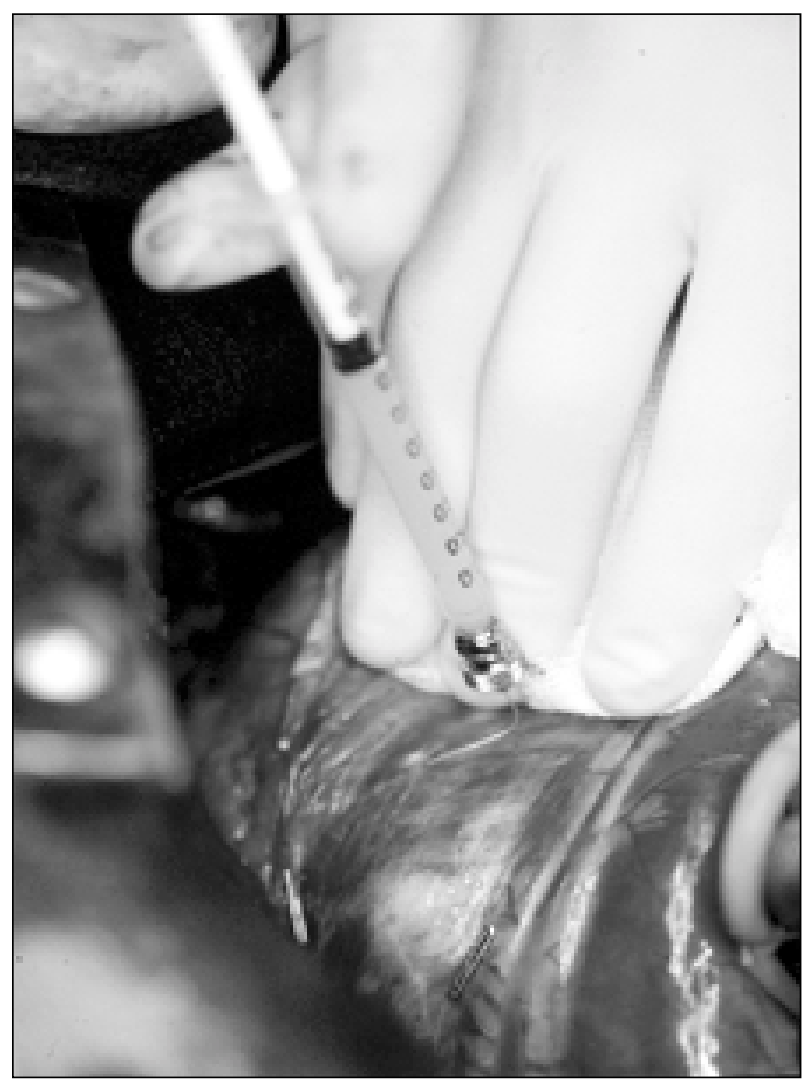

Fig. 1 - Myoblast transplantation in a patient with a 2-year-old myocardial infarction. Injection of culture medium containing $150 \times 10^{6} \mathrm{cells} / \mathrm{mL}$ into the infarcted area.

marked increase in the metabolism in the previously nonviable area demonstrated by PET.

Perhaps this functional improvement needs to be cautiously analyzed considering that 2 associated coronary artery bypass surgeries were performed on the left side of the heart. But the eminent fact of this report is the feasibility of a large-scale production of autologous myoblasts and their successful surgical engraftment, confirmed by the new-onset metabolic activity in the previously dead zone.

Many questions still need to be clarified, however. Do skeletal myoblasts become fatigue resistant? What is their life span after transplantation? Do they form any kind of coupling with host cardiomyocytes? Do they acquire a cardiac-like phenotype? Researchers are trying to answer some of these questions. It has been demonstrated that 2 to 7 weeks after transplantation, ${ }^{15}$ myoblasts from fastfiber isoforms convert into slow-twitch muscle, which is more resistant to fatigue. The fact that grafted myoblasts were able to convert the fiber isoform may indirectly indicate that they were contracting. However, if they do not form any cardiac-specific junction as shown in some experimental studies ${ }^{15,17}$, one possible hypothesis to explain this paradox is that they probably contract in reaction to a mechanical stimulus instead of the classic electric stimulation through intercalated disks.

Another important point to be stressed is the ideal number of cells to be transplanted. Based on an experimental study in rats ${ }^{19}$, a direct relationship was shown between the number of cells and the improvement in function. Considering the clinical case mentioned above ${ }^{18}$, it appears that the number of cells required to restore the viability of a medium-sized myocardial infarction (loss of 20-30\% of left ventricle mass) is approximately 1 billion cells, which take 2 weeks to grow in a myogenic-specific culture medium, thus precluding the use of this therapy in an emergency setting.

Finally, looking at heart failure natural history and the severity of the remodeling process intensified over time, the optimal period for patients to undergo cellular transplantation still needs to be determined. In all animal models, cellular transplantation has been used with success early after the myocardial infarction, before end- stage forms of heart dysfunction appear. Perhaps one of the main advantages of this therapy is to stop the left ventricular remodeling process, when performed in a medium- dilated left ventricle, allowing consequently the hypertrophy of the remaining viable cardiomyocytes in association with the new engrafted skeletal muscle to improve heart function.

In summary, it is important to emphasize the consistency of these experimental data. Regardless of the species ( rat $^{8}$, mouse ${ }^{9}$, rabbit ${ }^{16}$ ); the type of contractile cells (fetal cardiomyocytes ${ }^{7,16}$, skeletal myoblasts ${ }^{16,17}$ ); the model (coronary artery ligation ${ }^{18}$, cryonecrosis ${ }^{7}$, anthracycline-induced global cardiomyopathy ${ }^{9}$ ) and the assessment method (Langendorff perfusion ${ }^{7}$, sonomicrometry ${ }^{16}$, echocardiography ${ }^{6}$ ), cellular transplantation has consistently resulted in a definite improvement of function, which provides a strong rationale for the use of this technique in humans. The phase 1 clinical trial (in which 9 patients will be treated) is being undertaken in France with the aim of demonstrating the feasibility and the safety of the procedure. If no hazardous side effects are detected, then a large multicenter study will be initiated to assess the real place of this therapy in the heart failure therapeutic armamentarium.

\section{References}

1. Batista R, Verde J, Nery P, et al. Partial ventriculectomy to treat mid-stage heart disease. Ann Thorac Surg 1997; 64: 634-8.

2. Leriche R, Fontaine R. Essai expérimental de traitement de certains infarctus du myocarde et de l'anévrysme du coeur par une grffe de muscle strié. Bull Soc Nat Chir 1933; 9: 229-32.
3. Carpentier A, Chachques J. Myocardial stimulation with a stimulated skeletal muscle: first successfull clinical case (letter). Lancet 1985; 1: 1267.

4. Soonpaa MH, Koh GY, Klug MG, Field LJ. Formation of nascent intercalated disks between grafted fetal cardiomyocytes and host myocardium. Science 1994; 264: 98-101. 
5. Koh G, Soonpaa M, Klug M, et al. Stable fetal cardiomyocyte grafts in the hearts of dystrophic mice and dogs. J Clin Invest 1995; 96: 2034-2042.

6. Scorsin M, Marotte F, Sabri A, et al. Can grafted cardiomyocytes colonize periinfarction myocardial areas? Circulation 1996; 94: II-337-II-40.

7. Li R-K, JiaZ-Q, Weisel RD, et al. Cardiomyocyte transplantation improves heart function. Ann Thorac Surg 1996; 62: 654-61.

8. Scorsin M, Hagege A, Marotte F, et al. Does transplantation of cardiomyocytes im prove function of infarcted myocardium? Circulation 1997; 96(suppl II): 188-93.

9. Scorsin M, Hagege A, Dolizy I, et al. Can cellular transplantation improve function in doxorubicin-induced heart failure? Circulation 1998; 98: II-151-II-6.

10. Makino S, Fukuda K, Miyoshi S, et al. Cardiomyocytes can be generated from marrow stromal cells in vitro. J Clin Invest 1999; 103: 697-705.

11. Wang J, Shum-Tim D, Galipeau J, Chedrawy E, Eliopoulos N, Chiu R. Marrow stromal cells for cellular cardiomyoplasty: feasibility and potential clinical advantages. J Thorac Cardiovasc Surg 2000; 120: 999-1006.

12. Tomita S, Li R-K, Weisel R, et al. Autologous ransplantation of bone marrow cells improves dameged heart function. Circulation 1999; 100(suppl II): II-247-II56.
13. Mauro A. Satellite cell of skeletal muscle fiber. J Biochem Cytol 1961; 9:493-5.

14. Chiu RC-J, Zibaitis A, Kao RL. Cellular cardiomyoplasty: myocardial regeneration with satellite cell implantation. Ann Thorac Surg 1995; 60: 12-18.

15. Murry C, Wiseman R, Schwartz S, Hauschka S. Skeletal myoblast transplantation for repair of myocardial necrosis. J Clin Invest 1996; 98: 2512-23.

16. Taylor $D$, Atkins $B$, Hungspreungs $P$, et al. Regenerating functional myocardium: improved performance after skeletal myoblast transplantation. Nat Med 1998;4: 929-33.

17. Scorsin M, Hagege A, Vilquin J-T, et al. Comparison of the effects of fetal cardiomyocyte and skeletal myoblast transplantation on postinfarction left ventricular function. J Thorac Cardiovasc Surg 2000; 119: 1169-75.

18. Menasché P, Hagege A, Scorsin M, et al. Myoblast transplantation for heart failure. Lancet 2001; 357: 279-80

19. PouzetB, Vilquin J, MessasE, etal. Factors affecting functional outcome following autologous skeletal myoblast transplantation. Ann Thorac Surg 2001; 71: 844-51. 